\title{
INVESTIGACIÓN Y ÉTICA EN LA FORMACIÓN DE ESPECIALISTAS MÉDICOS EN CHILE: UN DEBATE PENDIENTE
}

\section{Introducción ${ }^{5}$}

La educación superior es considerada un faro de desarrollo científico, tecnológico y cultural desde el cual se proyecta la historia y el avance de una nación. Se concibe la misión de la universidad como la preservación de la riqueza cultural y formación de los futuros líderes mediante un sistema que privilegia la formación de competencias iluminadas desde el campo de la investigación científica, la enseñanza de calidad y la ética. Es dentro de este contexto donde se forjan las políticas educativas orientadas a perfeccionar a los médicos en Chile, tales como los Programas de Postítulo de Especialidades Médicas (PPEM).

El conjunto de universidades que forman médicos en Chile asumen desde mediados del siglo XX el desafío de ofrecer los PPEM. Su propósito es, por un lado, capacitar a los médicos cirujanos en las áreas primarias o derivadas de diferentes disciplinas médicas y, por otro, abrir espacios para el desarrollo de competencias para la investigación clínica en términos de una formación actual y éticamente sólida.

Se asume, en un primer aspecto, que la especialidad médica es ciencia porque su enseñanza se basa en la aplicación de los resultados de las evidencias científicas; es investigación porque requiere del cuestionamiento y análisis constante de los resultados, y es humanista ya que la relación médico-paciente es estrecha y personal (Csendes, 2007). Es precisamente en el entorno de los futuros especialistas donde se genera el ambiente académico propicio para la formación y estimulación de la investigación clínica. Un segundo aspecto que motiva investigar sobre la productividad científica clínica es que

5 Este estudio forma parte de un proyecto de investigación interdisciplinario financiado por el Consejo de Educación Superior. 
resulta primordial favorecer la formación de profesionales capaces de formular hipótesis clínicamente relevantes que orienten las acciones de salud. De no ser así, el beneficio que producen los nuevos conocimientos, medicamentos y tecnologías se retarda excesivamente (Stewart, 2003; Cech y col., 2001; Pober y col., 2001; Hotez, 2003; Salas y Rigotti, 2005). De ahí que el estudio de la productividad científica y formación ética de los médicos graduados de los PPEM durante el último decenio constituye un aspecto clave para evaluar la eficacia de las propuestas programáticas y, de esta forma, asegurar la calidad en salud. Si bien los programas han logrado consolidarse en el tiempo y cuentan con indicadores que certifican públicamente la calidad de los profesionales formados, son escasos los estudios que informan sobre el cumplimiento de sus metas e impacto en la productividad científica.

Este trabajo tiene por objetivo explorar las características de los PPEM en Chile, a partir de la información sistematizada disponible en siete universidades del país sobre el desarrollo de competencias en investigación clínica, formación ética y productividad científica de los graduados. Todo ello con la intención de contribuir a la discusión sobre la formación permanente de especialistas médicos y a la toma de decisiones respecto a los programas para potenciar su calidad. El estudio se justifica por el escaso número de investigaciones referidas a la productividad científica de dichos programas y es transversal dada la inclusión de la mayoría de las instituciones que los dictan en el país.

El presente artículo se estructura en cuatro ejes temáticos. El primero, antecedentes de los programas de postítulo, introduce el marco contextual y conceptual que está a la base del estudio, enfatizando el análisis teórico y pedagógico de los programas. El segundo, da cuenta de la metodología empleada, resaltando los procedimientos e instrumentos utilizados para dar rigor y validez a los datos. El tercero, dedicado a los resultados, presenta tablas y gráficos que sintetizan los principales hallazgos. Finalmente, se incluye la discusión y conclusiones, cuyo foco está en el cumplimiento de los objetivos propuestos y la contribución que la investigación hace al debate sobre tales programas, todo ello complementado con las 
referencias bibliográficas respecto del estado del arte en la temática abordada.

\section{Antecedentes de los programas de postítulo}

El propósito de los PPEM es formar médicos especialistas con sólida preparación científica en el campo clínico-asistencial, capacitados para programar y administrar acciones en salud en los diferentes niveles del ámbito social y administrativo del país. Las especialidades primarias ofrecidas corresponden, entre otras, a Anatomía Patológica, Cirugía, Medicina Interna, Medicina Familiar y Comunitaria, Obstetricia y Ginecología, Ortopedia y Traumatología, Pediatría, Psiquiatría Adultos, Urología, entre otras. Existen también programas de especialidades derivadas en Cardiología, Gastroenterología, Hematología, Nefrología, Oncología, Cirugía Plástica, por nombrar algunas, que se realizan después de aprobar una especialidad primaria. Los programas buscan cubrir las necesidades médicas crecientes en una población altamente demandante como la chilena.

El ingreso a los PPEM tiene como requisito la posesión de la Licenciatura en Medicina y el título profesional de Médico Cirujano. Las exigencias son comunes a todas las facultades de Medicina acogidas a la Asociación de Facultades de Medicina de Chile (ASOFAMECH). Los programas de especialización primaria tienen en general una duración de 36 meses y los de derivadas de 24 meses. Están organizados en asignaturas y actividades de aprendizaje especializadas (modulares), con un total de créditos que varía según la propuesta (por ejemplo, en la Universidad Austral de Chile, Anatomía Patológica exige un total de 145 créditos, mientras que Ortopedia y Traumatología 320). Los programas incluyen rotaciones por servicios hospitalarios, laboratorios y turnos de residencia asociados a la universidad formadora y por hospitales o centros de salud donde se realizan las actividades, pudiendo hacer parte de su formación en otros centros universitarios acreditados para dicho efecto.

Las facultades de Medicina que ofrecen PPEM están adscritas a las universidades de Chile, Pontificia Universidad Católica de Chile, La Frontera, Austral de Chile, Concepción, Valparaíso, Santiago de Chile, Los Andes, Mayor y Antofagasta. 
Una característica de los programas para especialistas médicos es la acreditación de los Centros Formadores (Facultades de Medicina) que los ofrecen. Esta política fue instaurada por ASOFAMECH para velar por la calidad de las instituciones formadoras, sus programas y sus egresados (ASOFAMECH, 1994). El proceso de acreditación tiene por objeto asegurar la formación de los especialistas y entregar al país profesionales con conocimientos teóricos, destrezas y actitudes que les permitan enfrentar con seguridad los procedimientos diagnósticos y terapéuticos propios de la especialidad (ASOFAMECH, 1994). En este contexto se analizan los programas y asignaturas, otorgándose particular importancia a la formación científica y ética que reciben estos profesionales. En 1979 se aprobó la creación de una Comisión Nacional de Acreditación de carácter técnico, con representantes de todas las facultades de Medicina (ASOFAMECH, 1994). Esta Comisión recibe las peticiones de acreditación formuladas por los centros docentes, las analiza mediante un comité de expertos que también realiza visitas en terreno y elabora informes específicos, emite pronunciamientos y dictámenes finales, examina apelaciones y renueva el proceso cada tres años. Adicionalmente, a partir de 1982, se realizan periódicamente seminarios de acreditación con la participación de decanos, representantes de escuelas de graduados y profesores especialistas de todas las universidades que dictan PPEM en Chile. La acreditación de los centros formadores es una acreditación académica, es decir, las facultades de Medicina garantizan que se reúnen las condiciones para formar especialistas idóneos. Este mecanismo de acreditación se fundamenta en el libre compromiso de los académicos y autoridades para someterse a él.

En síntesis, los PPEM responden a una necesidad social de grados crecientes de demanda, ofrecen a los médicos cirujanos la oportunidad de especializarse en áreas clínicas específicas y de vincularse con la comunidad científica mediante el desarrollo de competencias en investigación clínica en un entorno de valores éticos.

\section{Metodología}

La metodología es cuantitativa, exploratorio-transversal, bajo un diseño descriptivo no experimental y censal, lo que permite develar 
la inclusión de competencias en investigación clínica, actitudes éticas y la productividad científica de los graduados de los PPEM.

La población del estudio está constituida por los médicos graduados de programas de especialidades en el periodo 19992005. El diseño censal permite una mayor generalización de los resultados y evita el error por diseño muestral. Las unidades de análisis (programas de especialidad primaria o derivada, asignaturas y publicaciones) y los egresados se estratificaron según universidad y especialidad médica. Los criterios de inclusión de los programas fueron: a) consentimiento institucional para participar en el estudio; b) contar con egresados en el periodo de la investigación, y c) ofrecer las especialidades seleccionadas. Para la selección de estas últimas los criterios fueron: i) importancia médica y generación histórica del programa; ii) cobertura o tamaño de la población de graduados, y iii) balance entre programas del área médica y quirúrgica. El número de especialidades fue delimitado a seis primarias y seis derivadas, por razones prácticas y de profundización.

De acuerdo con los criterios de inclusión y selección, siete de las 10 universidades chilenas que ofrecen especialidades fueron incorporadas al estudio. Respecto de las tres universidades restantes, dos fueron excluidas por no contar con egresados en el periodo de estudio y una por decisión voluntaria de no participar. En orden a resguardar la confidencialidad, las universidades ingresadas fueron encriptadas bajo procedimiento de casos de estudio. Más adelante, la tabla 1 presenta los casos y las especialidades consideradas en la indagación.

Los programas de las especialidades primarias y derivadas seleccionadas, los contenidos de las respectivas asignaturas, las listas de alumnos egresados en el periodo 1999 y 2005 (ingresos 19972003) y las listas de profesores fueron facilitados por ASOFAMECH y las respectivas escuelas de graduados o direcciones de posgrado de cada facultad de Medicina participante. Los programas de tres de las universidades no correspondieron al periodo de estudio sino a los actualmente vigentes. 
Los instrumentos del estudio fueron: a) Lista de Cotejo de programas y mallas curriculares correspondientes, cuyo discurso pedagógico explícito fue valorado y conceptuado como regulador simbólico, consciente e inconsciente, de la selección, inclusión y desarrollo de competencias para generar habilidades científicas clínicas y actitudes éticas. Para ello se diseñó una subescala que permitió identificar presencia de un lenguaje descriptor de investigación clínica y actitudes éticas en los programas de cada asignatura, reconocible en el foco (título), propósitos o descripción, competencias y/u objetivos generales y específicos, contenidos, metodología, actividades, evaluación y bibliografía; b) Pauta de Base de Datos para la sistematización de la búsqueda de publicaciones generadas por los egresados entre 1999 y 2007, indexadas por el Institute for Scientific Information (ISI) y Scientific Electronic Library Online (SciELO). Para ello se consideraron todos los estudiantes ingresados entre 1997 y 2003 a las seis especialidades primarias y las seis derivadas seleccionadas. Debido a que la duración del periodo de estudios para una especialidad primaria es de tres años y una derivada dos años, la recopilación de los artículos se realizó entre 1999 y 2005, más dos años adicionales (2006 y 2007) por la tardanza normal en la aparición de las publicaciones. Tanto los instrumentos como los procedimientos utilizados fueron validados con juicio de expertos y depuración bajo criterios probabilísticos de duplicidad (doble digitación) y contrastación (comparaciones al azar).

Para la búsqueda de los artículos se accedió electrónicamente a las bases de datos ISI y SciELO. Los datos ISI fueron recopilados accediendo a la base de datos respectiva y empleando los filtros de búsqueda país, universidad y facultad de medicina definidos por la Biblioteca Central UACH. Estos datos fueron contrastados con una base de datos que contenía los nombres de cada alumno según universidad y año de egreso. A continuación los datos fueron filtrados usando las raíces de 54 descriptores de instituciones, especialidades y denominaciones médicas en español e inglés (clin, hosp, med, cir, hema, haem, etc.). Para ello se creó un programa en Visual Basic con un algoritmo que permitió formar las referencias de búsqueda, acceder a las bases de datos y generar nuevas bases acotada a los éxitos de búsqueda. Finalmente, los datos fueron revisados mediante 
comprobación visual para evitar la inclusión de errores aleatorios. A diferencia de la búsqueda ISI, la recopilación de las publicaciones SciELO se efectuó de manera selectiva utilizando como buscador el nombre de cada estudiante, generándose con los resultados archivos de planilla de cálculo. Se determinó el número de publicaciones efectivas, eliminándose las referencias cruzadas entre más de un estudiante.

Con el objetivo de acopiar elementos de análisis adicionales sobre la productividad científica de los programas de especialidades médicas, se examinó si los estudiantes estaban o no adscritos a proyectos de investigación concursables entre 1999 y 2007. Para ello se realizaron búsquedas en las bases de datos disponibles de FONDECYT, FONIS, FONDEF, INNOVA CHILE ${ }^{6}$ y de cada universidad seleccionada. Las búsquedas fueron contrastadas con una base de datos que contenía los nombres de cada alumno según especialidad, universidad y año de egreso. Los resultados obtenidos fueron acumulados en archivos de planilla de cálculo.

\section{Resultados}

En este apartado se muestran desde los análisis exploratorios hasta los descriptivos por variable, pasando por el comparativo relacionado con los casos o grupos.

Tabla 1. Universidades, programas de postítulos, estudiantes egresados y docentes en el periodo 1999-2005

\begin{tabular}{|c|c|c|c|c|}
\hline Universidad & $\begin{array}{c}\text { Número } \\
\text { programas }\end{array}$ & $\begin{array}{c}\text { Docentes } \\
\mathrm{N}(\%)\end{array}$ & $\begin{array}{c}\text { Estudiantes } \\
\mathrm{N}(\%)\end{array}$ & Relación docentes/estudiantes \\
\hline 1 & 7 & $131(6,0 \%)$ & $88(5,9 \%)$ & 1,5 \\
\hline 2 & 5 & $141(6,5 \%)$ & $23(1,5 \%)$ & 6,1 \\
\hline 3 & 10 & $232(10,7 \%)$ & $246(16,4 \%)$ & 0,9 \\
\hline 4 & 12 & $920(42,3 \%)$ & $748(50,0 \%)$ & 1,2 \\
\hline 5 & 6 & $186(8,5 \%)$ & $125(8,4 \%)$ & 1,5 \\
\hline 6 & 6 & $110(5,1 \%)$ & $80(5,3 \%)$ & 1,4 \\
\hline 7 & 10 & $456(21,0 \%)$ & $186(12,4 \%)$ & 2,5 \\
\hline Total & 56 & $2.176(100 \%)$ & $1.496(100 \%)$ & $2,1^{*}$ \\
\hline
\end{tabular}

* Corresponde a la media de relación docentes/estudiantes de las 7 universidades estudiadas.

6 Fondos concursables para investigadores en Chile. 
La tabla 1 presenta la información sobre las siete universidades estudiadas. Éstas ofrecen 56 programas correspondientes a los propuestos en esta investigación como representativos de especialidades primarias y derivadas. No todas ofrecen igual número de programas, situación que se explica por la tradicionalidad de algunas, lo que favorece su oferta, y la falta de suficientes capacidades instaladas, que impide ofrecer otros. Las universidades se pueden distribuir en dos grupos según cobertura de docentes y estudiantes: uno que comprende tres universidades que incluyen el 74\% de los docentes y el 78,8\% de los estudiantes (universidades 3, 4 y 7); otro, que aglutina cuatro universidades que comprenden el restante $26 \%$ de los docentes y $21,2 \%$ de estudiantes (universidades 1, 2, 5, y 6). Sólo con la excepción de las universidades 2 y 7 , el número de docentes y estudiantes posee un patrón de asociación más o menos estrecho; en los casos de excepción, es el número de docentes el que predomina. Este resultado determina que la media de la relación docente/estudiante sea 2,1. El número de docentes corresponde a número de sujetos y no a jornadas equivalentes.

Tabla 2. Distribución de estudiantes egresados por especialidad médica según universidad (1999-2005)

\begin{tabular}{|c|c|c|c|c|c|c|c|c|}
\hline \multirow{2}{*}{ ESPECIALIDADES } & \multicolumn{7}{|c|}{ UNIVERSIDAD } & \multirow{2}{*}{ Total } \\
\hline & 1 & 2 & 3 & 4 & 5 & 6 & 7 & \\
\hline \multicolumn{9}{|c|}{ PRIMARIAS } \\
\hline Medicina interna & 24 & 8 & 72 & 160 & 35 & 29 & 89 & 417 \\
\hline Pediatría & 21 & 3 & 69 & 189 & 30 & 17 & 20 & 349 \\
\hline Psiquiatría & 7 & 2 & 3 & 88 & 14 & 1 & 5 & 120 \\
\hline Cirugía general & 14 & 3 & 28 & 103 & 21 & 19 & 36 & 224 \\
\hline Obstetricia y Ginecología & 13 & 7 & 39 & 125 & 21 & 9 & 18 & 232 \\
\hline Urología & 6 & & 7 & 27 & 4 & 5 & 6 & 55 \\
\hline \multicolumn{9}{|c|}{ DERIVADAS } \\
\hline Endocrinología & & & 8 & 9 & & & 3 & 20 \\
\hline Nefrología & 3 & & 5 & 9 & & & 3 & 20 \\
\hline Gastroenterología & & & 7 & 17 & & & 5 & 29 \\
\hline Cirugía cabeza y cuello & & & & 6 & & & & 6 \\
\hline Cirugía cardiovascular & & & 8 & 4 & & & 1 & 13 \\
\hline Cirugía plástica & & & & 11 & & & & 11 \\
\hline Total & 88 & 23 & 246 & 748 & 125 & 80 & 186 & $1.496^{\circ}$ \\
\hline
\end{tabular}

* Se considera que puede existir un error de subestimación del 2,5\% en el número total de estudiantes. 
En la tabla 2 se observa la distribución de estudiantes egresados por especialidades primarias y derivadas en las siete universidades del estudio. Como se constata, las primarias tienen una mayor cobertura que las derivadas, lo cual responde a la lógica de que estas últimas son subespecialidades. En las primarias predominan, en orden decreciente, Medicina interna, Pediatría, Cirugía general, Obstetricia y Ginecología y Psiquiatría. La excepción es Urología, cuya cobertura se asemeja a las derivadas. En relación con este caso, una de las universidades del estudio la considera subespecialidad. A pesar del bajo número de estudiantes en las derivadas, las seleccionadas en este estudio, como Nefrología, Gastroenterología y Endocrinología y Cirugía cardiovascular reúnen el mayor número de sujetos. En cambio Cirugía plástica y Cirugía de cuello y cabeza sólo se dictan en una de las siete universidades en estudio, con cobertura docente-estudiante mínima. Consistente con lo analizado en la tabla 1, y con excepción de las especialidades de Cirugía general y Cirugía cardiovascular, el número de docentes y estudiantes por especialidad mantiene el equilibrio observado en la tabla 1 (información no mostrada).

A continuación se presentan los resultados del análisis de los programas de estudio y asignaturas de los PPEM respecto de la intención explícita de formación de competencias en investigación clínica y en actitudes éticas.

Gráfico 1. Distribución porcentual de programas por institución según intención explícita de formación de competencias en investigación clínica y actitudes éticas.

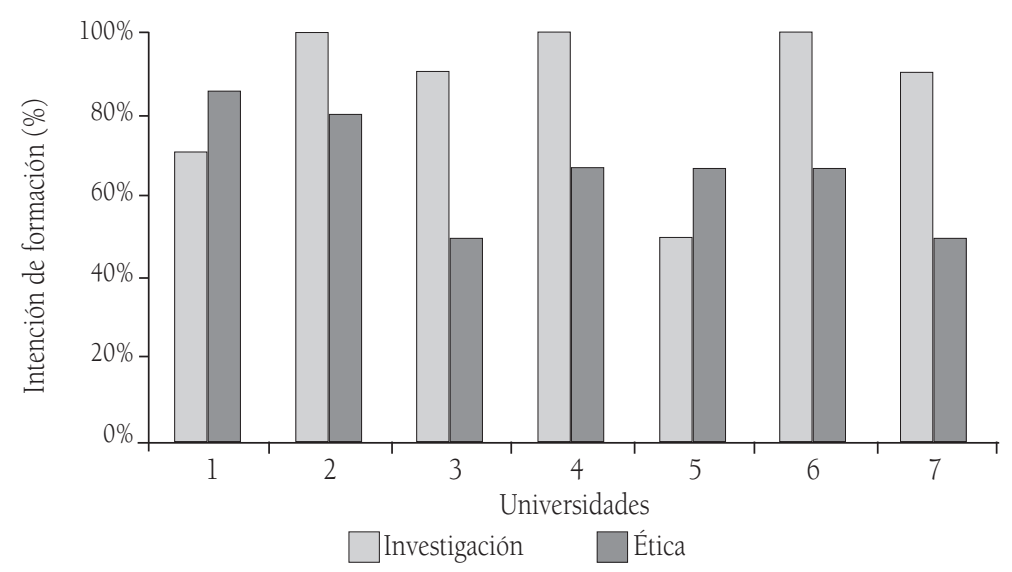


Gráfico 2. Distribución porcentual de asignaturas por institución según intención explícita de formación de competencias en investigación clínica y actitudes éticas.

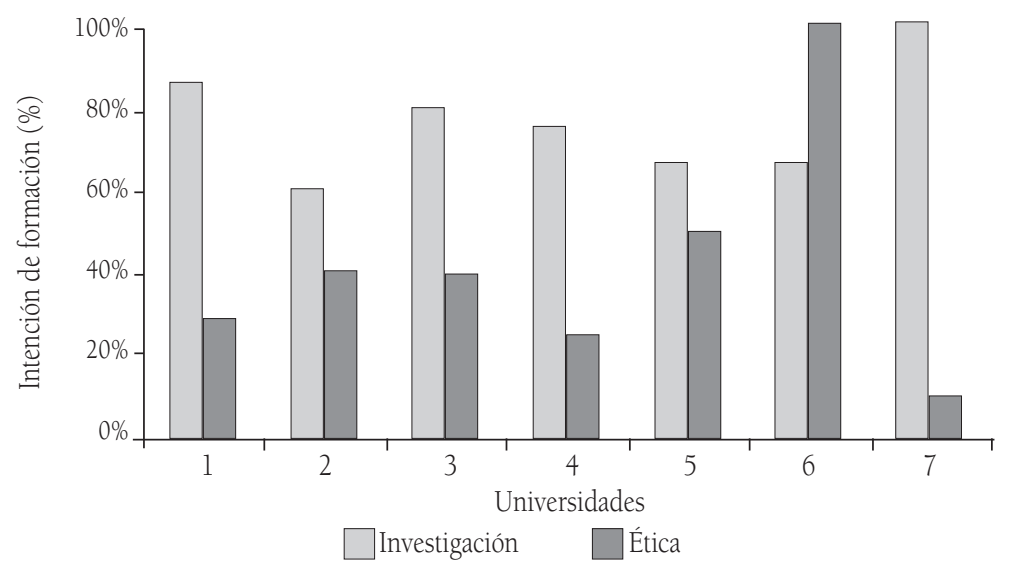

El gráfico 1 muestra que, en los programas que ofrecen las universidades objeto del estudio, hay una alta intención explícita de formación en competencias en investigación y, en menor medida, de actitudes éticas. Son excepción a esta regla las universidades 1 y 5 , en las que esta relación se invierte. El gráfico 2 analiza las asignaturas de dichos programas, revelando, al igual que el gráfico anterior, una preponderancia de la intencionalidad explícita de formación en competencias en investigación sobre las actitudes éticas, con la excepción de la universidad 6. Al comparar los programas (gráfico 1) con las asignaturas (gráfico 2) se observa que en estas últimas el desequilibrio es mayor entre la intención de formación en investigación y la formación de conductas éticas. El análisis de las asignaturas revela además una menor intencionalidad por formar a los estudiantes en ambas competencias. 
Tabla 3. Publicaciones ISI y SciELO según estudiantes por universidad (1999-2007)

\begin{tabular}{|c|c|c|c|c|}
\hline & \multicolumn{2}{|c|}{ ISI } & \multicolumn{2}{c|}{ SciELO } \\
\hline Universidad & $\mathrm{P}^{1}$ & $\mathrm{IP}^{2}$ & $\mathrm{P}$ & $\mathrm{IP}$ \\
\hline 1 & 13 & 0,15 & 20 & 0,23 \\
\hline 2 & 7 & 0,30 & 14 & 0,61 \\
\hline 3 & 264 & 1,07 & 268 & 1,09 \\
\hline 4 & 781 & 1,04 & 279 & 0,37 \\
\hline 5 & 21 & 0,17 & 56 & 0,45 \\
\hline 6 & 33 & 0,41 & 45 & 0,56 \\
\hline 7 & 16 & 0,08 & 79 & 0,42 \\
\hline Totales & 1135 & 0,76 & 761 & 0,51 \\
\hline
\end{tabular}

${ }^{1}$ Publicaciones por universidad.

2 Índice de publicaciones por estudiante (número de publicaciones/número de sujetos).

De acuerdo con la búsqueda realizada en las bases de datos bibliográficos ISI y SciELO, los estudiantes egresados de las especialidades médicas estudiadas en las siete universidades generaron en el periodo 1999-2007 menos de una publicación por sujeto (tabla 3). En efecto, la media de los índices de publicaciones de las siete universidades fueron 0,76 (ISI) y 0,51 (SciELO). Al desglosar las cifras anteriores, se observa que los valores ISI más altos corresponden preferentemente a las universidades 3 y 4 , las que se encuentran sobre la media nacional. En estas dos universidades el índice de publicaciones SciELO es similar al de ISI (universidad 3) o aún mucho menor (universidad 4). En cambio, las restantes universidades, que se caracterizan por una baja productividad ISI, presentan índices de publicaciones SciELO superiores al de ISI. La tabla 3 también muestra que la productividad ISI no está asociada al número total de estudiantes participantes en cada programa (compárese las universidades 1 y $6 ; 2$ y $7 ; 3$ y 4).

En general, se observó correspondencia entre el aspecto de las publicaciones ISI (título del artículo y/o área de la revista de publicación) y la especialidad cursada por el estudiante (información no mostrada). Sin embargo, la universidad 4 fue una excepción a esta correlación. En ésta un elevado número de publicaciones ISI no poseía relación temática con los contenidos propios del programa de especialidad del estudiante ( $55 \%)$.

El examen de las bases de datos FONDECYT, FONIS, FONDEF, INNOVA CHILE y de las siete universidades seleccionadas reveló que 
los estudiantes de PPEM están escasamente adscritos a proyectos científicos concursables. Estos correspondieron sólo a proyectos FONDECYT e institucionales propios de cada universidad. Respecto de éstos últimos, cabe hacer notar que los hallazgos de la búsqueda demostraron que las posibilidades de acceso a la información son desiguales debido a que no todas las universidades la han instalado en sus páginas web. Los resultados fueron los siguientes: universidad $1=$ 0 ; universidad $2=0$ (s/i de proyectos institucionales); universidad $3=$ 4 en FONDECYT, 2 en institucionales; universidad $4=9$ en FONDECYT (s/i de proyectos institucionales); universidad $5=1$ en FONDECYT, 3 en institucionales; universidad $6=0$; universidad $7=0$ (s/i de proyectos institucionales). Aunque las universidades 3 y 4 tienen una tendencia mayor a incorporar estudiantes de especialidades en proyectos de investigación, el número de incorporaciones en el periodo estudiado es exiguo (6/246 y 9/748, respectivamente).

\section{Discusión y conclusiones}

Este es un trabajo pionero en Chile, que analiza de manera sistemática y empírica las especialidades médicas en su intencionalidad formativa y la concreción de estas políticas en investigaciones clínicas realizadas por los estudiantes. Por otra parte, permite conocer cómo los programas de postítulo intencionan el desarrollo de actitudes éticas. Los resultados presentados tienen la importancia de abarcar siete de 10 universidades chilenas que ofrecen dichos programas.

Es evidente que los PPEM corresponden a esfuerzos formativos de carácter tutorial cuyo énfasis está en la relación formativa y personalizada docente-estudiante. La relación del número de docentes por estudiante encontrada es un reflejo de lo anterior. Sin embargo, el número de docentes debería corregirse por "jornadas equivalentes", porque es un hecho corriente en las universidades chilenas que los docentes clínicos, a diferencia de los académicos de otras áreas, poseen predominantemente contratos a jornada parcial. A pesar de ello, la relación docente-estudiante observada es similar a los programas de posgrado en las áreas de ciencias biológicas y en menor medida en las sociales. Este último factor introduce un nuevo elemento en la discusión acerca de la distinción realizada en Chile entre los programas 
de posgrado (magíster y doctorado; Ministerio de Educación, 2000) y los postítulos médicos. Por otra parte, y sin perjuicio que este estudio comprendió un grupo de especialidades primarias y derivadas representativas, la cobertura de los PPEM y el número de docentes y estudiantes egresados pueden considerarse como una respuesta que las universidades y los médicos egresados de los programas están dando a necesidades de salud de Chile.

La formación de competencias en investigación clínica y actitudes éticas corresponde a un elemento central de este estudio. En primer lugar, se evidencia una distinción entre programas y asignaturas respecto al énfasis que éstas le otorgan a ambos tipos de formación en su foco (título), objetivos, contenidos, metodología, actividades y evaluación. En tal sentido, el estudio interpreta que las asignaturas constituyen un indicador de mayor fidelidad con relación al desarrollo de ambas competencias. Estas evidencias podrían reflejar un "sinceramiento" en la declaración de acciones y actividades concretas -las asignaturasrespecto a la declaración de las políticas generales -los programas. Una probable explicación es que quienes formulan los programas de asignaturas ajustan los contenidos a su experiencia, las posibilidades concretas de realización de las acciones propuestas y el tiempo disponible para ello. En cambio, la formulación de un programa de estudio corresponde más bien a una declaración genérica de intenciones cuya operacionalidad queda supeditada a las asignaturas.

A diferencia de la percepción ética, que es de amplio uso, sería el grado de experiencia del equipo académico en investigación científica el que condiciona la concreción de lo propuesto en este campo. De un modo más general, la intención formativa de programas y asignaturas se desarrolla en el contexto de las respuestas a las políticas nacionales universitarias, las cuales enfatizan la necesidad de optimizar el componente curricular, de enmarcarlo en un cambio del paradigma educativo que privilegia el discurso pedagógico característico del enfoque por competencias y su ejercicio en documentos generales, tales como los programas de estudio. Esta situación se ve favorecida por mecanismos de aseguramiento de la calidad de la oferta formativa que destacan su análisis en el contraste del perfil de egreso con la capacidad institucional y la acción lógica documentada. El desafío 
pendiente es crear las herramientas necesarias para que el discurso se aproxime estrechamente a los objetivos que se proponen.

Nuestros resultados también revelan un énfasis en lo investigativo por sobre lo ético en ambas expresiones curriculares: programas y asignaturas. Este hecho puede ser interpretado como una muestra de la racionalidad pragmática que enmarca al sistema universitario de país, cuya expresión más fidedigna es el actual debate sobre el sistema de Aporte Fiscal Indirecto (AFI), en el cual el componente de "indagación" es pieza clave en el financiamiento y lo "ético" descansaría más bien en una exigencia de segundo orden, más disciplinaria que institucional. Es importante destacar, además, lo complejo que resulta aplicar la formación en actitudes éticas en el contexto universitario, tradicionalmente academicista y racional (Bernasconi y Rojas, 2003). Un ejemplo de ello es la relación inversa que evidencian los datos entre la formación en investigación y en actitudes éticas en las asignaturas. Una excepción notable a este resultado es la situación de la universidad 6 que podría estar reflejando la emergencia de un modelo distinto de formación. Sin cuestión de lo anterior, los 56 programas y sus asignaturas revelan, claramente, una elevada intencionalidad por la formación de actitudes éticas. Una hipótesis es que esta conducta está más relacionada con la necesidad de enfrentar valóricamente las presiones que diariamente afectan al médico en su desempeño profesional (de pacientes, empresas farmacéuticas, administrativas, entre otras.) que con la investigación clínica.

El número de publicaciones científicas generadas por los estudiantes de especialidades médicas es bajo - 0,76 publicaciones ISI por estudiante en un periodo de ocho años- como para asociar la alta intencionalidad en formación ética con las conductas esperables de futuros especialistas que también pueden emprender investigaciones clínicas. Se valora el desarrollo de investigación clínica éticamente responsable y, debido al progresivo aumento de esta clase de investigación, es recomendable una intensificación de la formación ética. Esta tendencia se refleja en los comités de ética creados en los principales hospitales públicos chilenos, los que actúan como entes reguladores del creciente aumento de estudios clínicos financiados por empresas farmacéuticas (Tunis y col., 2003; Carson, 2006). 
La productividad científica de los PPEM, medida en publicaciones científicas ISI y SciELO generadas por los estudiantes egresados puede ser considerada naciente. No obstante, debe considerarse que la corrección del índice de productividad por la varianza del número de publicaciones entre las siete universidades examinadas afecta a la baja dicho índice, debido a que dos de ellas (3 y 4) producen el 92\% de las publicaciones. En Francia, un médico ya ha producido entre dos y tres publicaciones indexadas ISI a su egreso de la especialidad de Dermatología (Concha M., observación no publicada). Ciertamente, las especialidades médicas corresponden a programas de formación profesional y, en adición, no es posible esperar que todos los especialistas médicos se interesen por la investigación científica. No obstante, esta última cumple una función esencial en la salud y prosperidad de una nación. Buxton y col. (2004) resumieron los beneficios que produce la investigación en salud como aquel "valor intrínseco" que posee la sociedad. Estos autores han señalado que el impacto de la investigación clínica se traduce en ahorro en costos para los sistemas de salud públicos y privados, incremento en la fuerza laboral sana, beneficios económicos producidos por el desarrollo de nuevos productos comerciales y en un amplío rango de beneficios sociales. En Chile la investigación clínica puede contribuir de manera importante a mejorar la efectividad y el costo-eficacia de los actuales tratamientos, innovar en las terapias y producir beneficios netos a la nación (Innova Chile, 2006).

De una manera amplia, se puede señalar que en un mundo caracterizado por una creciente tendencia a la globalización, cada país se ve en la necesidad de fomentar el potencial de su población y de elevar su nivel de productividad, conocimiento, flexibilidad, diversidad y apertura (Miranda, 2005). Simultáneamente, en la sociedad científica se evidencia un escenario de escisión entre el conocimiento científico y su puesta en práctica, cuyos efectos en el caso de la salud pretenden ser contrastados a través de propuestas de formación sistemáticas, tendientes a establecer un puente entre la ciencia básica y la aplicada (Friedrich, 2008). A pesar del impulso a la investigación clínica en particular y de las ciencias en general, en Chile la indagación científica ha sido una ocupación minoritaria: bien asentada en las ciencias naturales, creciente en las sociales y 
embrionaria en el área profesional. Tanto en la encuesta Carnegie de 1993 como en los estudios de Schiefelbein se constata el desinterés y la baja productividad de los académicos chilenos, entre los cuales un grupo minoritario (5\%) genera el 70\% de los artículos (Bernasconi y Rojas, 2003). La educación superior debe asumir el liderazgo académico para favorecer la educación y la investigación de calidad, funciones que deben realizarse bajo exigencias que garanticen el cumplimiento de parámetros internacionales (Lenn, 1997; Neave, 2002; CINDA, 2002; Lavados, 2003).

Chile tradicionalmente ha contado con adecuados estándares de práctica médica. El prestigio adquirido, asociado a los avances generales ocurridos en el país, atrae incluso a médicos extranjeros a formarse en el país. No obstante, Salas y Rigotti (2005) advirtieron que la sostenida disminución de la participación de médicos en proyectos de investigación científica es una realidad preocupante que merece análisis y soluciones en el corto plazo. Los resultados de la presente investigación amplían esta información, revelando una inicial productividad científica entre los egresados de los programas de especialidades médicas, medida en publicaciones ISI o SciELO. Es importante subrayar que los PPEM corresponden a un periodo de la formación clínica comparable con los magísteres o doctorados en el posgrado. La baja productividad observada contrasta notablemente con la elevada intencionalidad expresada en los programas por la formación de competencias en investigación clínica. Aunque si bien su inclusión como actividad formativa indica que la investigación es valorada, esta apreciación es insuficientemente concretada y debe despejarse la duda de que los esfuerzos existentes representen emprendimientos personales.

Una limitación del presente estudio es que no explica por qué dos universidades ( 3 y 4) se distancian del resto, generando más publicaciones. Aunque ambas exhiben un mayor número de estudiantes adscritos a proyectos de investigación concursables, este número es demasiado reducido para explicar la notable diferencia de productividad respecto de las restantes cinco universidades. La reducida adscripción de los estudiantes de PPEM a proyectos científicos concursables es efectivamente un problema común a las siete universidades estudiadas. Si bien algunos de los tipos de 
proyectos examinados se implementaron fuertemente sólo a fines o con posterioridad al periodo aquí estudiado (FONIS e INNOVA CHILE), es probable que el resultado obtenido se explique porque, de no existir políticas específicas en los programas, la transitoriedad del estudiante es un impedimento a su adscripción a proyectos. Por otra parte, nuestros resultados sugieren que el número de publicaciones no depende del número de estudiantes por universidad. Sin embargo, el alto grado de falta de correspondencia entre el asunto de las publicaciones ISI y la especialidad cursada, detectado en la universidad 4, marca una diferencia notable respecto de las restantes seis universidades. Al respecto, no es posible descartar una falla en la búsqueda electrónica, provocada por ejemplo por coincidencia de apellidos e iniciales con unos pocos investigadores de la misma facultad, altamente productivos. Su exacto significado debe ser aclarado en futuros estudios, porque se trata de una situación específica que involucra a una sola de las universidades estudiadas, notoriamente la de mayor productividad total.

La disminución de la productividad científica clínica se ha producido también en países desarrollados y ha merecido enérgicas respuestas paliativas, como las realizadas por el National Institute of Health en Estados Unidos de América entre 1997 y 1999 (NIH, 1997). La complejidad del problema amerita enfrentarlo con diferentes estrategias (Moskowitz y Thompson, 2001). Ejemplo de ello son el fortalecimiento de la investigación en el pregrado (Solomon y col., 2003; Hotez, 2003), lo cual implica asignar a las escuelas de medicina fondos especiales y realizar cambios curriculares que permitan alentar la práctica investigativa (Fang y Meyer, 2003; Swain, 1996). Otra posibilidad es incorporar cursos de investigación clínica y una tesis, ya sea directamente en los PPEM o por medio de la asociación del programa a un posgrado. La idea de elaborar una tesis de grado se basa en el reconocimiento que ésta constituye un mecanismo eficaz para producir cambios cuantitativos y cualitativos en la productividad científica y académica de una escuela o facultad y es una importante herramienta para la formación de nuevos cuadros académicos. Desde hace varios años y de manera progresiva las facultades de Medicina del país han creado programas de posgrado asociados a la investigación clínica. Independientemente de estas medidas, también algunas 
facultades incorporan cursos de investigación a los PPEM. Se puede señalar que todos estos esfuerzos requieren de la acción concertada de las autoridades que producen las políticas nacionales, las instituciones de apoyo a la investigación científica, autoridades académicas locales, entre otras. No obstante, a la luz de la eficiente tarea cumplida por ASOFAMECH y la Comisión Nacional de Acreditación en resguardo del profesionalismo de las especialidades médicas, es esperable que los cambios que se efectúan en el sistema de acreditación nacional de los posgrados y postítulos (Heerlein, 2008) contribuyan positivamente a alentar el fortalecimiento de la investigación clínica en Chile.

En conclusión, entre las dos variables consideradas en el presente trabajo -formación de competencias en investigación clínica y en actitudes éticas-la primera es la que tiene una intencionalidad mayor y la presencia más relevante en las dos universidades que más aportan a la productividad científica, expresada en publicaciones indexadas ISI y SciELO, situación que se relaciona con la tradicionalidad y racionalidad pragmática que caracteriza al actual sistema de educación superior del país. El hecho de que los estudiantes provenientes de universidades de menor presencia formativa y productividad científica publiquen más en revistas indexadas SciELO que ISI sugiere que, por un lado, los PPEM cumplen, aunque de manera restringida, su objetivo relativo a desarrollar competencias en investigación clínica y, por otro, que el modelo formativo personalizado y tutorial empleado en los programas tendría una influencia en los factores que inciden en la productividad científica y la transferencia del conocimiento a los pacientes.

Se agradece la ayuda en la recopilación de la información a las estudiantes de la Escuela de Periodismo de la Universidad Austral de Chile Teresa Martínez y Jessica Quijada; a Millaray Gavilán por su búsqueda bibliográfica, y a los estadísticos Gastón Vergara e Irma Molina, cuya colaboración fue fundamental para la concreción de este estudio.

\section{Referencias bibliográficas}

ASOFAMECH. (1994) $5^{\circ}$ Seminario Nacional de Acreditación de Centros Formadores de Especialistas. Santiago, Chile: ASOFAMECH. 
INNOVA CHILE. (2006) Biomedicina en Chile. Tendencias y oportunidades. Subdirección de Programas en Biotecnología. Santiago, Chile: INNOVA CHILE.

Bernasconi, A.; Rojas, F. (edit.) (2004) Informe Sobre la Educación Superior en Chile: 1980-2003. Santiago, Chile: Universitaria.

Buxton, M.; Hanney, S.; Jones T. (2004) Estimating the economic value to societies of the impact of health research: a critical review. Bulletin of World Health Organization, 82, pp. 733-739.

Carson, P.A. (2006) Clinical research in dermatology - 10 steps to getting started. Dermatologic Therapy, 19, pp. 377-382.

Cech, T.; Egan, L.; Doyle, C. et al. (2001) The Biomedical Research Bottleneck. Science, 293(5530), p. 573.

CINDA (2002) Indicadores universitarios: Experiencias y desafios internacionales de educación. Santiago, Chile: CINDA.

Csendes, A. (2007) Importancia de la investigación clínica en la formación de nuevos cirujanos. Revista Chilena de Cirugía, 59, pp. 171-182.

NIH (1997) Executive Summary-NIH Director's Panel on Clinical Research Report 12/97. Disponible en http://www.nih.gov/news/crp/97report/execsum. htm.

Fang, D.; Meyer, R. (2003) Effect of two Howard Hughes Medical Institute research medical training programs for medical students on the likelihood of pursuing research careers. Academic Medicine, 78, pp. 1271-1280.

Friedrich, M. (2008) A novel program seeks to take clinical scientists off the endangered species list. Journal of American Medicine Association, 29, pp. 1019-1020.

Heerlein, A. (2008) Acreditación de Programas de Formación de postítulo en medicina. Revista Médica de Chile, 136, pp. 937-938.

Hotez, P. (2003) Loss of laboratory instruction in American medical schools: erosion of Flexner's view of scientific medical education. American Journal of the Medical Sciences, 325, pp. 10-14.

Lavados, J. (2003) La educación superior chilena y los desafios de la globalización. Disponible en http://www.cse.cl/CSE/Asp/WEB_CSEpublic_pdf.asp.

Lenn, M.P. (2007) La Globalización de la educación superior: Temas Transnacionales de acceso, movilidad y calidad. Disponible en http:www.cse.cl/doc/WEB_ CSE public_peace.pdf.

Malcolm, W. (2003) Making sense of social research. London: Sage. 
Ministerio de Educación. (2000) Normas de Procedimiento para la Evaluación Experimental de la Calidad de los Programas de Postgrado de las Universidades Autónomas. Decreto Exento $N$ o3380. Santiago, Chile: Ministerio de Educación.

Miranda, Ch. (2005) Formación permanente de profesores. Impacto en sus competencias profesionales. Santiago, Chile: Pontificia Universidad Católica de Chile.

Moskowitz, J.; Thompson, J.N. (2001) Enhancing the Clinical Research Pipeline: Training Approaches for a New Century. Academic Medicine, 76, pp. 307-15

Naciones Unidas (1997) Educating for a sustainable future: A transdisciplinary vision for concerted action. In: Neave, G. (2002) Research and ResearchTraining Systems: Towards a Typology. Disponible en http://unesdoc. unesco.org/images/0011/001106/110686s.pdf.

Pober, J.; Neuhauser, C.; Pober J.A. (2001) Obstacles facing translational research in academic medical centers. Federation of American Societies of Experimental Biology Journal, 15, pp. 2303-2313.

Salas, S.; Rigotti A. (2005) Médicos-científicos en Chile: ¿Una especie en extinción? Revista Médica de Chile, 133, pp. 121-28.

Solomon, S.; Pichert T.; Wasserman D. (2003) Impact of medical students research in the development of physician-scientists. The Journal of Investigative Medicine, 51, pp. 149-156.

Sean, R.; Tunis, MD.; Daniel B. et al. (2003) Practical clinical trials: increasing the value of clinical research for decision making in clinical and health policy. Journal of American Medicine Association, 290, pp. 1624-632.

Stewart, P. (2003) Improving clinical research. British Medical Journal, 327, pp. 999-1000.

Swain, J. (1996) Presidential Address to the American Society for Clinical Investigation. Washington, DC. May 4, 1996. Is there room left for academics in academic medicine? Journal of Clinical Investigation, 98, pp. 1071-1073.

UNESCO (1997) Educating for a Sustainable Future: A Transdisciplinary Vision for Concerted Action. UNESCO. Disponible en: http://www.unesco. org/education/tlsf/TLSF/theme_a/mod01/uncom01t05s01.htm

Recibido: 29 de septiembre de 2008

Aceptado: 4 de noviembre de 2008 